\title{
Epileptic encephalopathy with global cerebral demyelination
}

INSERM

\section{Source}

INSERM. (1999). Orphanet: an online rare disease and orphan drug data base. Epileptic encephalopathy with global cerebral demyelination. ORPHA:353217

Epileptic encephalopathy with global cerebral demyelination is a rare mitochondrial substrate carrier disorder characterized by severe muscular hypotonia, seizures (with or without episodic apnea) beginning in the first year of life, and arrested psychomotor development (affecting mainly motor skills). Severe spasticity with hyperreflexia has also been reported. Global cerebral hypomyelination is a characteristic imaging feature of this disease. 